\title{
De la "ciudad eterna" a las "misiones más remotas": comunicación epistolar de los generales con los jesuitas de Sinaloa, 1591-1630
}

\author{
"The Eternal City" to "The Most Remote Missions": Epistolary Communications from the
} Generals to the Jesuits in Sinaloa, 1591-1630.

Gilberto López Castillo*

\section{Resumen}

El seguimiento de una fuente -el Epistolario del General de la Compañía de Jesús- permite realizar una caracterización de las comunicaciones entre las misiones norteñas de la Provincia Mexicana y el centro de la orden en Roma. Así, las cartas publicadas en Monumenta Mexicana y su complemento en el Archivum Romanum Societatis Iesu posibilitan que se tenga un punto de vista más completo del proceso de establecimiento de la misión en Sinaloa desde su concepción durante el generalato de Claudio Acquaviva, hasta la etapa final de Mutio Vitelleschi. El epistolario es una fuente privilegiada, sin embargo de integrarse sólo por las cartas enviadas desde Roma a la periferia, en virtud que ofrece un marcapasos de los avances y retrocesos de la evangelización, de la interacción entre los actores sociales de este proceso y de los cambios en los mecanismos de administración jesuítica. Al entrecruzar esta fuente con otras de la administración civil, así como con la bibliografía sobre el tema nos encontramos un panorama más completo de esta etapa fundacional de una de las empresas que marcaron la presencia de los jesuitas en Nueva España, sobre la que tradicionalmente ha privado el punto de vista de los actores locales.

\footnotetext{
* Profesor-investigador del Instituto Nacional de Antropología e Historia (INAH), en Sinaloa, México. Obtuvo el doctorado en Ciencias Sociales por la Universidad de Guadalajara (México) fue becario posdoctoral en el Instituto Universitario Europeo bajo la dirección de Antonella Romano (2010-2011), uno de cuyos frutos es el presente artículo. Es miembro del Seminario la religión y los jesuitas en el Noroeste Novohispano y del Seminario de Instituciones novohispanas.
} 


\title{
Palabras clave
}

Provincia Mexicana, Claudio Acquaviva, MutioVitelleschi, Misiones, Roma.

\begin{abstract}
By analysing the collected correspondence of the Generals of the Society of Jesus, we can develop an overall picture of the communication between the northern missions of the Mexican Province and the centre of the Society in Rome. As such, the letters published in the Monumenta Mexicana and their complement in the Archivum Romanum Societatis Iesu permit a more complete vision of the process of the establishment of the Sinaloan mission, from its conception during the generalcy of Claudio Acquaviva through to the final stages of the generalcy of MutioVitelleschi. The correspondence of the Generals of the Society is a valuable source - notwithstanding the limitations of being one-way correspondence from Rome to the Provinces - as it indicates the successes and failures of the process of evangelisation, the interaction between social actors within this process and the changes in the mechanisms of Jesuit administration. By comparing this source with others from the civil administration, together with the historiography of the theme, we find ourselves with a more complete panorama of this foundational stage of one of the enterprises that marked the presence of the Jesuits in New Spain-one which traditionally has been deprived of the perspective of local actors.
\end{abstract}

\section{Key words}

Mexican Province, Claudio Acquaviva, MutioVitelleschi, Missions, Rome.

\section{Recibido: 18 de diciembre de 2013}

Evaluado: 17 de enero de 2014 


\section{Nuestra fuente}

La introducción del Epistolario del General para el estudio de la Compañía de Jesús en Nueva España proviene del trabajo de Félix Zubillaga, tanto al editar las transcripciones de los documentos del epistolario en Monumenta Mexicana, como al utilizar ampliamente su contenido en las introducciones que acompañan los volúmenes bajo su responsabilidad ${ }^{1}$.

En conjunto, el Epistolario contiene las respuestas que los generales daban a las cartas recibidas de cada uno de los miembros de la orden, en el caso que nos ocupa tanto de los provinciales de México, como de los superiores de Sinaloa y de jesuitas en lo individual, que se han conservado en los registros de cartas de los generales Claudio Acquaviva (1581-1615) y Mutio Vitelleschi (1615-1645). Es pues una fuente que cubre toda la Provincia Mexicana de la Compañía de Jesús y de la consulta del conjunto se utiliza la información específica de nuestro interés ${ }^{2}$.

Como fuente de información histórica, las cartas de los generales se suman a otras como las annuas, los informes y las crónicas que nos permiten investigar aspectos del último cuarto del siglo XVI y buena parte del siglo XVII. Son fuentes que se complementan entre sí, y que nos ofrecen un amplio panorama del trabajo de los jesuitas en el orbe. El epistolario incluye una gran variedad de temas, por ejemplo para el caso de las misiones de Paraguay Martín Morales, quien ha trabajado este fondo de forma sistemática en el análisis y edición documental correspondiente al primer tercio del siglo XVII encontró que las misivas van desde las relaciones con los obispos y gobernadores, la vida espiritual, la formación, la participación de los jesuitas en los conflictos bélicos, el castigo a los indios y diversos aspectos de la vida religiosa ${ }^{3}$. Por su parte Carlos A. Page también ha desarrollado esta labor mediante la publicación de las cartas de los generales Tirso González, Miguel Ángel Tamburini y F. Retz, con lo que ha logrado ofrecer un seguimiento puntual a la historia del colegio jesuita de Córdoba, de la misma provincia del Paraguay, tocante a la disposición de los espacios, la actividad económica y la construcción de los templos de la orden $^{4}$.

\footnotetext{
${ }^{1}$ Zubillaga, 1956, 1959, 1968, 1971, 1973, 1976 y 1981. Posteriormente se publicó un volumen más: Rodríguez, 1991.

${ }^{2}$ Archivum Romanum Societatis Iesu (en adelante ARSI), Fondo México, Epistolario del General (Epp. Gen.), Vols. 1, 2 y 3. En virtud de que la serie Monumenta Mexicana sólo publicó documentos correspondientes al periodo 1572-1605, en esta ocasión también se ha consultado el resto del periodo de Claudio Acquaviva correspondiente al Epistolario del General, que termina en 1615 y gran parte del correspondiente a Mutio Vitelleschi, que va de 1616 a 1645. Para un análisis de la documentación de Monumenta Mexicana ver Castañeda y Gruzinski, 1978: 106-131. El mismo grupo documental relativo a la Provincia Mexicana de la Compañía de Jesús incluye cartas de los generales Everardo Mercuriano (1572-1580), Juan Pablo Oliva (1664-1681), Carlos de Nolleye (1682-1686) y las del comienzo del generalato de Tirso González (16871705).

${ }^{3}$ Morales, 2005: 23 y 45.

${ }^{4}$ Page, 1999: 5-24 y 2013: 248-321. La importancia de la obra de este autor es que mientras otros hemos utilizado esta fuente desde su origen en la Curia Generalicia en el ARSI, él lo realiza a partir de las cartas que con el mismo nombre existen en Argentina referidas a la Provincia del Paraguay lo que nos da una idea de la importancia de estos conjuntos documentales, celosamente guardados en cada una de las provincias jesuíticas para el gobierno de la orden.
} 
Para el caso de las misiones del norte novohispano que eran también un ámbito de frontera, la temática es muy similar y refiere a estrategias misioneras y nuevos campos de evangelización, economía interna de las misiones, residencias y colegios, regulación de la vida cotidiana, relaciones con las autoridades locales y regionales, rebeliones, casos de intercesión, discrepancias en la administración religiosa, consuelo a los misioneros, así como temas de escándalo.

El sistema de comunicaciones de la Compañía de Jesús no estaba exento de controversias y en algún sentido había la incredulidad de muchos jesuitas que permanecían fuera de las estructuras internas de gobierno de la orden, respecto de que realmente el general mismo en Roma hiciera caso de sus misivas, o de que tomaran en cuenta sus puntos de vista de frente a las opiniones de superiores, no siempre coincidentes, e incluso de que se filtrara su punto de vista respecto de sus compañeros. Ante una de estas inquietudes Claudio Acquaviva contestó al Padre Antonio Rubio sobre la materia:

V.R. sepa que yo veo las cartas de los que no gobiernan con la mesma voluntad que las de los superiores. Y admito lo que se me escribe, de muy buena gana. Aunque dar a cada persona y cosa su lugar, claro es que ha de quedar a mi cargo; y también, resolver lo que se huviere de hacer; porque la luz y noticia que acá se tiene de todo, crea V.R. que es más copiosa que la de ningún particular sujeto ni superior. Ansí que V.R. y qualquiera otro puede escribirme, siempre que dello se consolaren; y esto, sin otro rezelo, sino que en mi hallarán todo el consuelo y ayuda que yo les pudiera dar.

Se trata, sin duda de un tema recurrente, sin embargo los rumores de cualquier tipo llegaban tarde o temprano hasta Roma y la posibilidad de que todos los jesuitas pudieran escribir directo al general ofrecía una rica perspectiva de los diversos asuntos, como se expresa en este testimonio de 1591, aún en los principios del generalato de Acquaviva. Nuevas expresiones de este tipo llegaron al general en 1607, donde la desconfianza de algunos miembros de la orden los llevaba simplemente a prescindir de este sistema de comunicaciones, de lo que Acquaviva se lamentaba en virtud de que se trataba de uno de los elementos clave para el gobierno de las distintas provincias de la Compañía. ${ }^{6}$

\section{Las cartas relativas a las misiones de Sinaloa}

Para esta investigación se consultaron las cartas que tenían que ver con la "misión de Sinaloa", así como de la vecina de Guadiana (Topia, Tepehuanes y Parras inclusive), cuando la temática refería a las dos, o había referencias explícitas a Sinaloa. Ello debido a que usualmente la información que tiene que ver con las áreas misionales novohispanas se

\footnotetext{
5 “Acquaviva, al Padre Antonio Rubio, Roma 10 de junio de 1591”, ARSI, Mex. 01, Epp. Gen.: 103 y v. Normalmente las fechas con que se cuenta sólo refieren al año, si bien, cuando sea posible se incluirá también el día y el mes, así como el establecimiento o el nombramiento del jesuita u otra persona a quien vaya dirigida la correspondencia.

6 “Acquaviva, al Padre Pedro de Morales, Roma, 15 de enero de 1607”, ARSI, Mex. 02, Epp. Gen.: 74.

${ }^{7}$ Utilizamos la palabra Sinaloa, con letra "s" de acuerdo a la forma que ha perdurado, sin embargo, durante los siglos XVI y XVII se escribía con "c" o con "z". Sólo cuando se trata de una cita textual se respeta la ortografía original, que usualmente fue con "c".
} 
abordaba por los generales en grupo, sin que ello obste para que gran parte de la correspondencia tenga que ver con aspectos específicos de la administración misional al ras del suelo, tocante a una residencia, un colegio o una misión. Como sea, ello impedirá que realice en esta ocasión algún tipo de trabajo estadístico, sino que más bien la idea es que a partir de un caso se puede destacar los aspectos relevantes de esta fuente para la historia de la Compañía de Jesús.

Tenemos dos tipos de cartas, en primera instancia las dirigidas por los generales a los misioneros en lo individual, al superior, o al rector de Sinaloa (cuando este fue erigido como colegio incoado en 1616), o a individuos en específico de fuera de la orden; también aquellas que se enviaban a algún jesuita en funciones de visitador. El otro tipo de cartas contenía mayor información en tanto que se dirigía a los provinciales de Nueva España y eran una especie de instrucciones que habrían de desarrollarse en los distintos campos de trabajo de la Compañía -no sólo en las tierras de misión-. Solía ocurrir que en estas cartas se apuntara detalladamente los problemas expuestos por los operarios en lo individual, de tal forma que correspondía al provincial supervisar la solución de los problemas.

Las primeras referencias romanas sobre Sinaloa provienen de la comunicación entre los superiores -particularmente el provincial Pedro Díaz y el visitador Rodrigo de Avellaneda- y el general, aunque no provienen en todos los casos del Epistolario del General. Así, Avellaneda refería del provincial:

Tiene un bordón [un estribillo]: que ay poca gente para missiones; y certifico a V.P. que es engaño; que, antes, por tenerlos todos ociosos, no están con el consuelo que ternían[tendrían] si se ocupasen; y con los diez que van, ahora, al 3 año de probación, repartiéndolos, como yo pienso repartirlos, o enviar otros más antiguos quedándose ellos, o algunos de ellos, en lugar déstos, según que fuesen más a propósito de las missiones, por los collegios que tiene, tan pocos como Pátzcuaro, Valladolid y Guadalajara, embiándolos de dos en dos, me sobrarán otros dos que embiar a la Cinaloa con el Padre Gonzalo de Tapia; y otros dos a la Topia sobre que me escribe un capitán, cuya copia va con ésta, o al Nuevo México, si se descubriese, como el virrey espera cada día ${ }^{8}$.

Se abría entonces para la Compañía de Jesús un amplio campo de trabajo entre los indios, que era promovido desde Roma por Acquaviva y que fomentó ampliamente el visitador Avellaneda. A medida que se adquiría la experiencia entre los diversos grupos indígenas de la "tierra adentro", los jesuitas se convencieron de los problemas que traería consigo secularizar misiones pasando los primeros años del proceso evangelizador, como preveía la legislación. Así, el Padre Pedro Díaz en su carácter de provincial, expresó su preocupación al general y pidió su disposición puntual tocante a la perspectiva de tener que entregar "la misión de Cinaloa" a clérigos seglares al consolidar las fundaciones entre los indios, pues desde su punto de vista ello significaría "totalmente perderlos", ; y si bien no

\footnotetext{
8 "El Padre Diego de Avellaneda, visitador, al Padre Acquaviva, Tepotzotlán, 25 de septiembre de 1591" en Zubillaga (ed.), 1971: 63.

9 "El Padre Pedro Díaz [provincial México] a nuestro Padre Acquaviva, México, 12 de mayo de 1592", ARSI, Mex. 16, Historia 1565-1600: 70v.
} 
contamos con la respuesta específica, es claro que ello no ocurrió, al menos durante los siglos XVI y XVII ${ }^{10}$.

Es importante destacar que la relación directa entre Gonzalo de Tapia y el general Claudio Acquaviva respecto de Sinaloa aconteció en agosto de 1592, a partir de una carta enviada por el misionero. Es, propiamente, la presentación de la empresa evangelizadora iniciada un año antes por el mismo Padre, en su carácter de superior junto con el Padre Martín Pérez. Queda claro en primera instancia que habían sido destinados por el visitador de la Provincia Mexicana a una "misión entre infieles donde pareciese convenir" "11 y que fue el gobernador de la Nueva Vizcaya Rodrigo del Río Loza quien, tras su arribo a Guadiana (capital de la Nueva Vizcaya), decidió enviarlos a Sinaloa, un territorio costero en el ámbito de su jurisdicción. Además de la información geográfica y etnográfica relativa a los grupos de indios cahitas, Tapia se expresó de uno de los temas que se volvieron recurrentes en las comunicaciones de los generales y los superiores en la Provincia Mexicana, como fue la aptitud de los jesuitas enviados a misión, desilusionado del actuar de los primeros refuerzos en Sinaloa. Era importante que quienes fueran destinados a misión tuvieran idea de las circunstancias a que se enfrentarían, de tal forma que expresó las condiciones en cuanto a la austeridad, la distancia y la soledad: "en estas tierras no hay vestido que mudar, no hay casa en qué vivir, no hay quien aderece de comer, ni muchas veces qué aderezar",

Las referencias correspondientes a las primeras dos décadas de la misión de Sinaloa son escasas, de tal forma que la respuesta de Acquaviva a Tapia es la que abre el Epistolario del General directamente con Sinaloa:

El consuelo que Dios saca de los fructuosos trabajos de V.R. no se queda en México: que acá nos alcanza buena parte y nos da no pequeña ocasión de alabar y adorar la divina misericordia que, con tanta liberalidad, se va comunicando entre esa ciega gentilidad. $Y$, cierto, Padre mío, que aunque yo siento lo mucho que V.R. $y$ vuestros compañeros forzosamente padecerán en tierras y entre gentes tales: todavía, creo que les tengo más envidia que compasión por que es felicíssima suerte la de aquellos que Dios toma por instrumentos para descubrirse a esa pobre y desamparada gente; y no dudo de su piadosa bondad, sino que, quanto sus operarios más viven solos ay, y más desnudos de los humanos entretenimientos que, en otras ocupaciones, no faltan, tanto más les acudirá él, y les proveerá los divinos, que son de más utilidad y consuelo para aliviar los trabajos. Yo he encargado y, de nuevo encargo a los superiores que ayuden esa misión, porque se me trasluce que, por ella, se va abriendo una gran puerta para multiplicar la grei de Christo y manifestar su santísimo nombre, de manera que sea servido y adorado donde, hasta aora, no ha sido conocido ${ }^{13}$.

\footnotetext{
${ }^{10}$ López Castillo, 2010.

11 "El Padre Gonzalo de Tapia [superior, Cinaloa] al Padre Claudio Acquaviva, Cinaloa, 1 de agosto de 1592”, ARSI, Mex. 16, Historia 1565-1600: 107-108v.

12 Loc. cit.

13 “El Padre Claudio Acquaviva, al Padre Gonzalo de Tapia, Roma, 9 de marzo de 1594” en Zubillaga (ed.), 1973: 201-202.
} 
Había entonces la conciencia de que Sinaloa, así como los otros frentes de "misión entre infieles" abiertos por la Compañía -Guadiana, Zacatecas, La Laguna, San Luis de la Paz- le darían al fin la justificación moral tocante a su presencia en Nueva España, después de casi dos décadas en que se habían consolidado en las principales ciudades del virreinato. Durante esa época los testimonios insisten en la importancia de la actividad jesuítica entre los nativos y de hecho Zubillaga encuentra que "la recomendación de esta labor es el tema más recurrente en las cartas del Padre Acquaviva al provincial novohispano",14.

Ahora bien, el hecho de que haya pocas cartas del general a los jesuitas en Sinaloa refleja simplemente que había pocos misioneros -usualmente cuatro y un coadjutor durante la primera década- y que en esa etapa de inicios de la evangelización el tema del apoyo a la misión se trataba en otras escalas de la administración imperial y jesuítica, con menos énfasis en los actores locales ${ }^{15}$. En una carta de 9 de mayo de 1594 el general expresaba al visitador Avellaneda cómo a partir de su comunicación con Gonzalo de Tapia, se habrían enviado de Europa misioneros con el procurador Padre Pedro de Morales, si bien por testimonios alternos sabemos que eran 18 jesuitas para Sinaloa y otros 18 para Filipinas ${ }^{16}$. Incluso el establecimiento de una residencia de la Compañía en Guadiana, aprobada en 1595 por el general, se da en virtud del apoyo que daría a lo de Sinaloa ${ }^{17}$.

Tras la muerte violenta del Padre Tapia a manos de los indios el 11 de julio de 1594, de la que Acquaviva se enteró por carta del provincial Esteban Páez, el Epistolario del General registra de forma escueta la respuesta de Roma relativa a la información que ha recibido sobre el tema a partir de marzo de $1595^{18}$. Sin embargo, en años posteriores (al menos 1597 y 1607) la figura del Padre Tapia y su sangre derramada se convierten en un elemento de fortaleza en el discurso del general que buscaba ofrecer ánimo a la presencia de los misioneros. Así, al dirigirse al Padre Méndez expresaba que:

La que de V.R. he recevido, escrita el año de 96 me ha consolado mucho, entendiendo por ella lo que también me escriben otros que es el fructo que se hace en la missión de Zinaloa, y esperanças que hay de que, cada día será más copioso [...] Bien creo que ayudan y no poco las oraciones del buen Padre Tapia, las quales serán gran parte para que los que en aquella mies trabajaren, cojan el fructo que el pretendió con costa de su sangre [...]Raçón es que esa missión sea muy favorecida y ayudada, como creo lo será, del Padre provincial, según lo que el

\footnotetext{
${ }^{14}$ Ibíd.: 24.

${ }^{15}$ Por ejemplo hay cartas del virrey Luis de Velasco al monarca, "Luis de Velasco, el joven, virrey, a Felipe II, México, 6 de abril de 1594", en Ibíd.: 218-221, donde se destaca el elogio del gobernador Río de la Loza, en cuanto al trabajo de los jesuitas en Sinaloa; o del monarca a sus oficiales reales tocante a la forma en que ha de garantizarse el viaje de los jesuitas desde España a las misiones de Topia, Sinaloa y La Laguna, y su avituallamiento, "Felipe II, Rey a la Casa de Contratación de Sevilla, Madrid, 6 de abril de 1694" en Ibíd.: 213-215.

16 “Acquaviva, a Rodrigo del Río Loza [gobernador, Nueva Vizcaya], Roma, 9 de mayo de 1594” en Ibíd.: 244.

17 “Acquaviva, al Padre Martín Peláez [Cinaloa], Roma, 13 de marzo de 1595” en Ibíd.: 354-355.

18 “Acquaviva al Padre Esteban Páez [provincial, México], Roma, 13 de marzo de 1595” en Ibíd.:365-369.
} 
mesmo me escribe. Y fuéralo más si tuviéramos tanta abundancia de sujetos, como deseamos para enviarles socorro ${ }^{19}$.

Para entonces se ha abierto la comunicación directa con los demás miembros de la misión de Sinaloa. Por ejemplo en 1596, ésta se da en primera instancia con el Padre Martín Pérez, compañero que había sido del Padre Tapia, así como con el Padre Martín Peláez. Nos encontramos nuevamente con un tema que ya ha esbozado Tapia desde 1592 y 1593, como es, la falta de operarios que aparece en los dos testimonios, así como la notificación de haber recibido de Peláez "particular relación de lo que es aquella provincia, y de la grande puerta que de allí se va abriendo al evangelio",20.

\section{Hacia una consolidación de los establecimientos misionales}

Los años siguientes de la década tras su consabido costo en sangre para la Compañía son de incertidumbre, así como de búsqueda de la consolidación de los avances en cuanto a la evangelización en torno a la villa de San Felipe y Santiago de Sinaloa. Cabe decir que el riesgo en que se encontraban los misioneros era percibido por las autoridades virreinales que hicieron llegar al monarca información sobre la materia, de tal forma que incluso antes de la muerte del primer superior de Sinaloa se había previsto desde Madrid el apoyo militar para aquel establecimiento. Asimismo, se gestionó en distintas escalas de la administración imperial la aprobación para el repoblamiento de la villa de San Juan de Carapoa, en que originalmente se dio la ocupación hispana de la región. Los jesuitas mismos fueron gestores y promotores de aquella villa. Así por ejemplo, las primeras dos referencias correspondientes a los años 1601 y 1602 tienen que ver con el visto bueno que ofrece Claudio Acquaviva para que a petición de los Padres -en este caso quien se comunicaba sobre el tema era Hernando de Villafañe- se estableciera una nueva villa que "sería muy útil para las cosas de la tierra y para acudir con mayor seguridad a los ministerios sagrados" 21 . La citada villa y la función que se esperaba de ella como baluarte de los grupos cahitas del río Zuaque fue aprobada por el virrey hasta 1605, construyéndose un Fuerte que fue culminado en 1610 para la defensa del poblamiento hispano y que tomó el nombre de Villa de San Juan Bautista o Fuerte de Montesclaros ${ }^{22}$.

Luego hay un salto que nos lleva hasta 1609, en que se inicia una cierta regularidad en la comunicación epistolar con una, dos y hasta nueve cartas por año en los momentos más álgidos. Uno de los aspectos que registra esta fuente refiere al problema de conciencia que provocaba entre algunos religiosos que la residencia de Sinaloa tuviera una "estancia de ganado". Un primer punto de vista sobre su actividad económica proviene de 1597, al ser notificado el general, quien dispuso que se tuviese allí "solamente lo necesario para el

\footnotetext{
19 “Acquaviva, al Padre Pedro Méndez [Cinaloa], Roma, 2 de agosto de 1597” en Zubillaga (ed.), $1976: 272$. También “Acquaviva al Padre Pedro Méndez, Roma, 1607”, ARSI, Mex. 02, Epp. Gen.: 95v.

20 “Acquaviva, al Padre Martín Pérez [Cinaloa], Roma, 8 de abril de 1596” en Zubillaga (ed.), 1976: 130-131" y “Acquaviva, al Padre Martín Peláez [Cinaloa], Roma, 8 de abril de 1596” en Ibíd.: 131-132. Es evidente que los 18 jesuitas que acompañaban al procurador no llegaron a Sinaloa.

21 “Acquaviva, a Hernando de Villafañe [Cinaloa], abril 16 de 1601” y “abril 15 de 1602”, ARSI, Mex. 02, Epp. Gen.: 5v. y 20 respectivamente.

${ }^{22}$ López Castillo, 2009: 107-125.
} 
comer y el sustento, poco más o menos ${ }^{, 23}$. En 1614 insiste tocante a que la citada estancia pudiese mantenerse siempre cuidando de que fuera para satisfacer las necesidades de la administración religiosa ${ }^{24}$. Es un tema con diversas implicaciones, el primero es que en 1616, ya con el nuevo general, Mutio Vitelleschi se da la aceptación del establecimiento como "colegio incoado de Sinaloa", y que por tanto se le permite continuar con sus propiedades, pues así se garantizaría su permanencia ante la inexistencia de un "fundador" ". El tema de las propiedades del Colegio de Sinaloa continuó como uno de los aspectos problemáticos que se trataban en las cartas en buena parte del siglo. Podríamos decir que ya en 1668, es innegable el papel de las tierras del Colegio para la economía misional $^{26}$.

También ha quedado constancia de los diversos nombramientos para la administración de las misiones y la existencia de un "superintendente de las misiones", o de Padres visitadores, así como de los cambios que hubo en los primeros tiempos en esta materia. Las provincias de misión en Nueva España eran en primera instancia un grupo que tenía ciertas características que las diferenciaban claramente de los otros campos de trabajo de los jesuitas. Así, entre 1609 y 1614 hay diversas acepciones, la primera nos habla de la necesidad de un "superintendente para las misiones de la tierra adentro", en 1611 se da el nombramiento a Hernando de Villafañe como "superintendente de las misiones de la Nueva Vizcaya y Cinaloa"27. En 1619 tenemos un "superintendente general" que lo era nuevamente el Padre Hernando de Villafañe y su expresión de una necesidad específica que reflejaba el sentir de otros Padres como Andrés Pérez de Ribas que le habían escrito sobre lo mismo: la visita del Padre provincial a Sinaloa al menos una vez durante su periodo. Las cartas de Villafañe y Pérez debieron ser de 1618. Éste último, en pleno trabajo entre los yaquis recibió el visto bueno de Vitelleschi quien expresó sobre la materia que:

lo de visitar el Padre provincial estas misiones, va a ser de importancia y así se lo ruego y encargo apretadamente, pues es justo que los hijos de la Compañía que con tanto ejemplo están trabajando en ellas sean consolados y alentados del superior general $^{28}$.

$\mathrm{Al}$ año siguiente Vitelleschi le respondía que buscaría que ello se realizara, a la vez que daba instrucciones sobre la materia al Padre Nicolás de Arnaya, procurador general de

\footnotetext{
23 “Acquaviva al Padre Esteban Páez [provincial, México], Roma, 4 de agosto de 1597” en Zubillaga, 1976: 293.

24 “Acquaviva al Padre Rodrigo de Cabredo [provincial, México], Roma, 1614”, ARSI, Mex. 02, Epp. Gen., 166v. Ver López Castillo, 2007: 183-201.

25 “El Padre Mutio Vitelleschi al Padre Rodrigo Cabredo [provincial, México], 1616, asiento de los nuevos superiores de las casas y colegios de esa provincia", ARSI, Mex. 02, Epp. Gen.: 183v. Ver también Alegre, 1958: 248 y 629-630. Para el caso de Guadiana ver Pacheco, 2004: 53-59.

26 “Tirso González, general, al Padre provincial de México, 1668”, ARSI, Mex. 02, Epp. Gen.: 2-2v. Un amplio ensayo sobre el tema relativo al periodo anterior a las misiones en Zubillaga, 1969: 3-169. También López Castillo, 2008 y Faria, 1981.

27 “Acquaviva al Padre Hernando de Villafañe, Roma, 1612”, ARSI, Mex. 02, Epp. Gen.: 146v.

28 "Vitelleschi al Padre Andrés Pérez [Cinaloa], Roma, 1619”, ARSI, Mex. 02, Epp. Gen.: 211v. y "Vitelleschi al Padre Hernando de Villafañe, visitador general de Cinaloa, Roma, 1619”, ARSI, Mex. 02, Epp. Gen.: 211v.
} 
México $^{29}$. La visita a Guadiana debió realizarse en 1619 , no obstante en 1620 seguía como un tema pendiente del provincial la visita de Sinaloa ${ }^{30}$. El sistema de comunicación era en este sentido muy eficiente, ya que los temas expuestos por los misioneros, tenían una respuesta normalmente en el lapso de un año, aunque podría llevarse hasta dos. Las respuestas dependían del tipo de peticiones, la administración misional era la que tenía la mayor prioridad, si bien los Padres buscaban expresar por este medio otro tipo de necesidades. Por ejemplo el Padre Pérez de Ribas solicitó permiso para el envío de plata a su hermana que era monja, mientras que el Padre Francisco de Alinaro solicitaba sin éxito el envío de alguna imagen de Nuestra Señora ${ }^{31}$.

Durante la tercera década del siglo XVII se percibe una menor intensidad en las comunicaciones entre el general y los jesuitas de Sinaloa. Podríamos decir que para este momento la provincia de Sinaloa ha logrado su mayor extensión y que entró en un proceso de consolidación de los propios establecimientos, de tal forma que las relaciones de los Padres con el general disminuyen, con excepción de los misioneros del Yaqui, en pleno proceso de evangelización ${ }^{32}$.

Para esta década nos encontramos con algunas cartas en las que el general contesta tocante a individuos que le han sido recomendados en lo individual por servicios especiales a la Compañía por medio de algún misionero, o por comunicación directa de los interesados con el general. Por ejemplo, en el caso del capitán del presidio de Sinaloa, Diego Martínez de Hurdaide hubo comunicación directa del militar en 1621, así como la intercesión del Padre Juan Castiani que tuvo respuesta en 1622 y en 1625, año éste en el que el general consigna haberlo encargado a los sacerdotes de Madrid $^{33}$.

El otro caso nos lleva a una concesión especial para la madre del jesuita Hernando de Tovar. Éste había muerto como mártir en la rebelión tepehuana de $1616^{34}$ habiendo su madre, Isabel de Tovar, ingresado como monja al monasterio de San Lorenzo de la ciudad de México con el nombre de "Isabel de San Bernardo". La comunicación de Isabel fue directa con el general, si bien previamente había intercedido por ella el Padre Hernando de Villafañe. El trato especial solicitado y que el general concedió implicó que la monja

\footnotetext{
29 "Vitelleschi al Padre Nicolás de Arnaya, procurador general de México, Roma, 1619”, ARSI, Mex. 02, Epp. Gen.: $214 \mathrm{v}$.

30 "Vitelleschi a Nicolás de Arnaya, provincial de México, Roma, 1620”, ARSI, Mex. 02, Epp. Gen.: 231.

31 "Vitelleschi al Padre Francisco de Alinaro [o Alinario], Roma, 1625”, ARSI, Mex. 02, Epp. Gen.: 310.

${ }^{32}$ Pérez de Ribas, 1992: 283-357.

33 "Vitelleschi al Padre Alberto Clerici [Cinaloa], 1625", ARSI, Mex. 02, Epp. Gen.: 310. También "Vitelleschi al capitán Diego Martínez de Hurdaide, Sinaloa, 1622", ARSI, Mex. 02, Epp. Gen.: 267v. Los jesuitas tuvieron un eficiente sistema de procuración de sus intereses frente a otros actores, así mismo ofrecían sus buenos oficios con generosidad a sus benefactores y a los funcionarios de los que esperaban obtener un favor. En el caso de Sinaloa los problemas se trataban en Guadiana y Parral, tocante al gobierno de la Nueva Vizcaya; en la audiencia de Guadalajara, cabeza del reino de la Nueva Galicia - de la que estaba supeditado el gobierno de la Nueva Vizcaya- o directamente con el virrey en la ciudad de México. Sobre el funcionamiento de los procuradores del clero secular ver Mazín, 2007.

${ }^{34}$ Sobre el tema hay un estudio clásico: Gutiérrez Casillas, 1981. Más recientemente Redden, 2012: 101-128, en plan comparativo con Paraguay.
}

130 Gilberto López Castillo. De la "ciudad eterna" a las "misiones más remotas"... 121-136. 
pudiera recibir la confesión de los jesuitas, a pesar de que una de las instrucciones dadas con regularidad les prohibía brindar este tipo de servicios a las religiosas ${ }^{35}$.

Sin embargo, el trabajo con los indios, central en la tarea jesuítica aparece desdibujado en esta fuente, acaso se consignan las nuevas entradas, por ejemplo al río Yaqui por los Padres Andrés Pérez de Ribas y Tomás Basilio; la percepción que sobre los mismos tenía el Padre Francisco Alinaro en cuanto a que "los indios de ese partido son muy belicosos y por esta causa muy difíciles de tratar", a pesar de lo cual en tres años (1620) se habrían bautizado 7.000 personas; noticias sobre haber finalizado la desunión de los acaxees y xiximes por el Padre Andrés Tutino, o las buenas nuevas enviadas por el Padre Alinaro en su trabajo entre los nebomes en $1625^{36}$.

También hay referencias sobre documentos mayores. Por ejemplo, el Padre Martín de Brujes había reportado que después de estar en Sinaloa se encontraba en Manila, desde donde le habría mandado su "Relación de las causas que le pasaron en Cinaloa" o una “Lengua xixime” por el Padre Andrés Tutino, desde las misiones de Topia ${ }^{37}$.

Asimismo se reconocía que si bien el trabajo jesuita en las misiones era uno de los más dignos de la Compañía, también era preciso considerar el bien que hacía a los misioneros descansar de este tipo de trabajos. Por ello se recomendaba que quienes tuvieran necesidad de ello, pudiesen pasar a alguna de las residencias y colegios donde tuvieran un mejor clima y hubiera asimismo posibilidades para la atención de su salud. Parece ser que fueron muchos los que optaron por este descanso, de tal forma que ya en 1620 se indicaba al provincial que dispusiera lo necesario para que aquellos Padres, muchos con gran experiencia en las misiones volvieran a su labor directa con los indios, acción que de paso se convertiría en un ejemplo para los nuevos jesuitas ${ }^{38}$. Y es que el contraste con la realidad fue muy duro, para jesuitas como el Padre Juan Ángelo Balestra, quien misionaba en el río Mayo en 1623 y que propuso dejar la Compañía por la incompatibilidad del trabajo misional con sus expectativas de vida. Así lo caracterizaba Vitelleschi a partir de lo dicho por el propio misionero:

El gran trabajo con los indios no le permite tener tanto tiempo como quisiera para atender a sí mismo. Al pedirle permiso para cambiarse a la religión de los

\footnotetext{
35 "Acquaviva a Isabel de San Bernardo, religiosa en el monasterio de San Lorenzo de México, Roma, 1621", ARSI, Mex. 02, Epp. Gen.: 247v.- 248. Sobre la biografía de Isabel de Tovar ver Bazarte Martínez, Velázquez Soto y Cuéllar Zazueta, 2007: 15-49. Sobre las prohibiciones específicas se puede consultar "Respuestas romanas a la congregación provincial mexicana, celebrada en México, del 21 al 29 de enero de 1592, dadas en Roma en enero de 1594", en Zubillaga (ed.), 1973: 63.

36 "Vitelleschi al Padre Andrés Pérez, Roma, 1619", ARSI, Méx. 02, Epp. Gen.: 211v; "Vitelleschi al Padre Francisco Alinaro, Roma, 1621", ARSI, Mex. 02, Epp. Gen.: 254v; "Vitelleschi al Padre Andrés Tutino, Roma, 1612", ARSI, Mex. 02, Epp. Gen.: 146v. y "Vitelleschi al Padre Francisco de Alinaro, Roma, 1626", ARSI, Mex. 02, Epp. Gen.: 310.

37 "Vitelleschi al Padre Martín de Brujes, Roma, 1622", ARSI, Mex. 02, Epp. Gen.: 271 y "Acquaviva al Padre Andrés Tutino [Topia], Roma, 1614", ARSI, Mex. 02, Epp. Gen.: 167v.

38 "Respecto de la conveniencia de que el Padre San Clemente salga de Topia, 1614", ver "Acquaviva, al provincial de México, Rodrigo de Cabredo", ARSI, Mex. 02, Epp. Gen.: 166; "Acquavivaal Padre Juan Bautista Velasco [Sinaloa], Roma, 1614", ARSI, Mex. 02, Epp. Gen.: 164 y "Sobre lo mucho que el Padre Martín Peláez trabajó en estas misiones y la consideración de su posible retorno", ver "Acquaviva, al Padre Juan Bautista Velasco, [Cinaloa], 1613”, ARSI, Mex. 02, Epp. Gen.: 157.
} 
carmelitas descalzos le encarga al Padre provincial que lo ponga en puesto y ocupación más a propósito para todo lo que desea ${ }^{39}$.

De hecho no era la primera comunicación de Balestra sobre el tema, pues ya en 1619 daba testimonio de que entre sus perspectivas tenía al trabajo misionero sólo como algo temporal, asimismo de la necesidad de tener un compañero jesuita para su actividad evangelizadora $^{40}$. Pero no solo se daba el caso de jesuitas que pedían salir de su actividad entre los indios, sino que otros tuvieron que salir por órdenes del superior general debido a la búsqueda de corregir el ánimo de algún Padre, como el de Alonso Ruiz "por la aspereza de su condición”, o por no respetar reglas específicas, tales como la prohibición de enviar dinero a Europa, regla que infringió el jesuita Hernando de Santarén. ${ }^{41}$

En este sentido destacamos el carácter de excepción del Colegio de Sinaloa debido a que se trataba de un colegio en tierra de misiones, donde no había un grupo permanente de jesuitas, sino solo el rector y quizá algunos coadjutores ${ }^{42}$. El colegio en este sentido brindaba un alivio a los misioneros en tanto punto de reunión y de realización de ejercicios espirituales con el resto de los miembros de la provincia de San Felipe y Santiago. Ello era cotidiano, sin embargo el verdadero descanso implicaba salir del territorio de misión y dirigirse a uno de los colegios citadinos que ofrecieran otro tipo de clima, de ambiente y convivencia $^{43}$.

La primera mitad de la década de 1620 está marcada por el inicio de conflictos en el Colegio de Sinaloa, incluso entre el rector, Hernando de Villafañe y el capitán del presidio, Diego Martínez de Hurdaide. De hecho hubo varias cartas de misioneros que expresaron su inquietud sobre la materia, por ejemplo el Padre Blas de Paredes hacía patente "la falta de unión y charidad en Cinaloa",44, y el mismo Villafañe al retomar la rectoría expresaba que encontraba "algunas cosas de cuidado y muchas de consuelo"45. En realidad los temas de conflicto se reflejan mejor en la comunicación con el provincial de México, Padre Juan Lorencio y tienen dos vertientes, la primera, los trabajos de infraestructura hidráulica que se habría propuesto realizar Villafañe y que comprometían los recursos del colegio y la segunda el comercio que ordinariamente realizaban los Padres en las misiones. Así instruía al provincial en 1625 para que remediara lo que trataba de hacer el Padre Villafañe:

No falta quien me escribe que el Padre Hernando de Villafañe trataba de sacar un río en Cinaloa en que sería menester gastar más de 9000 ducados que serían la

\footnotetext{
39 “Vitelleschi, al Padre Juan Ángelo Balestra[Cinaloa], Roma, 1619”, ARSI, Mex. 02, Epp. Gen.: 258.

40 "Vitelleschi al Padre Juan Ángelo Balestra, 1619”, ARSI, Mex. 02, Epp. Gen.: 223.

41 "Vitelleschi al Padre Martín Peláez, viceprovincial de México, Roma, 1609”, ARSI, Mex. 02, Epp. Gen.: $124 \mathrm{v}$.

${ }^{42}$ En la residencia de Sinaloa y posteriormente en el colegio durante las primeras tres décadas de presencia jesuítica sólo estuvo el hermano coadjutor Francisco de Castro quien habría sido llevado por el Padre Gonzalo de Tapia, Pérez de Ribas, 1992: 235.

${ }^{43}$ Un amplio estudio en el que se desarrolla la idea del antiguo territorio misional, en este caso de Sonora, como un espacio de excepción: Almada Bay, 2008: 203-238.

44 "Vitelleschi, al Padre Blas de Paredes [Cinaloa], Roma, 1623”, ARSI, Mex. 02, Epp. Gen.: 295v.

45 "Vitelleschi, al Padre Hernando de Villafañe, [rector, Cinaloa], Roma, 1624”, ARSI, Mex. 02, Epp. Gen.: $295 \mathrm{v}$.
}

132 Gilberto López Castillo. De la "ciudad eterna" a las "misiones más remotas"... 121-136. 
ruina de aquel colegio. V. R. se informe de lo que hay en esto y ponga en ello conveniente remedio y encargue usted al dicho Padre que se componga y tenga paz. con el capitán Diego Martínez de Hurdaide, a quien debemos, según me dicen, muy buenas obras ${ }^{46}$.

Para resolver estos problemas se dispuso el envío de una persona "de mucho celo" a la visita de las misiones de Sinaloa, San Andrés, Topia, Tepehuanes y Parras:

Pues aunque hay unos misioneros muy siervos... otros hay, según me informan que tienen nombre de mercaderes porque a título de sus iglesias venden y compran y poseen cantidad de plata y dineros y muchas mulas y ganado mayor de lo cual resulta ser desestimado nuestro ministerio de las personas que ven tales deshorbitancias... ${ }^{47}$.

Los dos temas que mencionamos, el inicio de conflictos entre jesuitas y militares y la actividad de los religiosos que trascendía las labores de evangelización son aspectos que continuarán presentes durante el siglo XVII y del que estos datos son apenas las primeras muestras.

\section{Conclusiones}

Finalmente cabe decir que el Epistolario del General tiene una relevancia particular y es el hecho que cubre el primer medio siglo de la presencia jesuítica en Sinaloa, época de la que en efecto tenemos gran información, principalmente en las cartas annuas, pero también de la obra clásica del jesuita Andrés Pérez de Ribas, Triunfos de nuestra santa fe entre las gentes más bárbaras y fieras del Nuevo Orbe, de tal forma que al ofrecernos una perspectiva alterna levanta la cortina respecto del funcionamiento interno de una institución y ratifica detalles de la organización vertical de ejercicio del poder desde Roma hasta las provincias misionales "más remotas", y las interrelaciones entre unos y otros puntos. También nos ayuda a fechar el origen de ciertas actividades, tales como el desarrollo de la ganadería, la participación de los jesuitas en actividades comerciales y en fin, aspectos de la vida cotidiana y de los sentimientos individuales de estos hombres que, en algunos casos, se ven enfrentados a un destino que han buscado con pasión, mientras que otros lo han tenido que tomar debido a la regla de la obediencia a la que se han comprometido desde su ingreso a la corporación. Se trata pues de una fuente que se complementa con el resto de los documentos de la época, tanto jesuíticos como de la administración civil y se convierte en un marcapasos de los ritmos de la evangelización, y de los logros y problemas de la Compañía, que nos muestra simultáneamente la importancia de la misión de Sinaloa en el contexto del desarrollo de otros proyectos misionales en América. Es, en última instancia para los Padres misioneros, la oportunidad para expresarse y hacer que su voz se escuche en el centro de la orden, cuya caja de resonancia no siempre hacía eco a las necesidades individuales de los misioneros, pero que al menos les dejaba constancia de que formaban parte de una corporación global y de que su trabajo era relevante. Así, Vitelleschi podría

\footnotetext{
46 "Vitelleschi, al Padre Juan Lorencio, provincial de México, Roma, 1625”, ARSI, Mex. 02, Epp. Gen.: 322v.

${ }^{47}$ Loc. Cit.
}

133 Gilberto López Castillo. De la "ciudad eterna" a las "misiones más remotas"... 121-136. 
decirle a Olinaro que no había ocasión para la imagen solicitada de nuestra señora, pero que al menos lo dejaba "edificado, consolado y contento por su trabajo"

\section{Referencias}

\section{Archivos}

Archivum Romanum Societatis Iesu,

Fondo México, Epistolario del General (Epp. Gen.), Vols. 1-3.

Mex. 16, Historia 1565-1600

\section{Bibliografía}

Alegre, Francisco Javier (1958), Historia de la provincia de la Compañía de Jesús de Nueva España. Tomo II. Roma: Institutum Historicum Societatis Iesu (IHSI).

Almada Bay, Ignacio (2008), "Ilícitos, solidaridades y tradiciones locales en la construcción de una identidad territorial en la frontera norte de México. Sonora ¿una tierra de excepción? La perspectiva de antiguo régimen”. En Sariego Rodríguez, Juan Luis (Comp.), El norte de México. Entre fronteras. Chihuahua: INAH, pp. 203-238.

Bazarte Martínez, Alicia, Velázquez Soto, Idolina y Cuéllar Zazueta, Rina (2007), "Isabel de Tovar: viuda, musa y monja profesa”. En Alicia Bazarte Martínez, Desde el claustro de la Higuera. Objetos sacros y vida cotidiana en el ex convento Jerónimo de San Lorenzo. México: Instituto Politécnico Nacional, pp. 15-49.

Castañeda García, Carmen y Gruzinski Serge (1978), "Monumenta Mexicana. Los documentos de la Compañía de Jesús en la Nueva España. Siglo XVI”. Historia Mexicana 113, pp. 106-131.

Faria, Francisco Xavier de (1981), Apologético defensorio y puntual manifiesto. Culiacán: Universidad Autónoma de Sinaloa.

Gutiérrez Casillas, José (1981), Mártires jesuitas de los tepehuanes. México: Ed. Tradición.

Hausberger, Bernard (1997), "La vida cotidiana de los misioneros jesuitas en el noroeste novohispano". Estudios de Historia Novohispana 17, pp. 66-105.

López Castillo, Gilberto (2007), "El Colegio Jesuita de Sinaloa: recuento historiográfico y documental". En Pacheco, José de la Cruz, Vallebueno Garcinava, Miguel y Zazueta Manjarrez José Carlos (Coords.), Seminario la religión y los jesuitas en el Noroeste Novohispano, memoria volumen II. Culiacán: El Colegio de SinaloaUniversidad Juárez del Estado de Durango, pp. 183-201.

48 “Vitelleschi al Padre Francisco de Olinaro, Roma, 1625”, ARSI, Mex. 02, Epp. Gen.: 310. 
(2008), "El Colegio jesuita de Sinaloa -Nueva España- y su participación en el mercado de la tierra". En Memoria de las XII Jornadas Internacionales sobre las misiones jesuíticas, Buenos Aires: CONICET.

(2009) "Orígenes de la ocupación española del antiguo Fuerte de Montesclaros". En López Castillo Gilberto et. al. (Coords.), El patrimonio histórico y arqueológico del antiguo Fuerte de Montesclaros. México: INAH-Universidad Autónoma de Sinaloa-Ayuntamiento de El Fuerte, pp. 107-125.

(2010), El poblamiento en tierra de indios cahitas. Transformaciones de la territorialidad en el contexto de las misiones jesuitas 1591-1790. México, Siglo XXI Editores-El Colegio de Sinaloa.

Mazín, Oscar (2007), Gestores de la Real Justicia. Procuradores y agentes de las catedrales hispanas en la corte de Madrid. México: El Colegio de México.

Morales, Martin M. (Ed.) (2005), A mis manos han llegado. Cartas de los Padres generales a la antigua provincia del Paraguay 1608-1639. Madrid-Roma: Universidad Pontificia Madrid-IHSI, Monumenta Historicum Societatis Iesu, nueva serie.

Ortega Noriega, Sergio (2003), Un ensayo de historia regional, el Noroeste de México, 1530-1880, México: UNAM.

Pacheco, José de la Cruz (2004), El colegio de Guadiana de los jesuitas, 1596-1767. México: Universidad Juárez del Estado de Durango-Plaza y Valdés, 2004.

Page, Carlos A. (1999), "Córdoba y los jesuitas en las cartas de los padres generales dirigidas a los PP. de la Provincia Jesuítica del Paraguay. T. González, M. A. Tamburini y F. Retz (escritas entre 1696 y 1739)", Revista de la Junta Provincial de Historia de Córdoba, No. 17, pp. 5-24.

(2013), “Las cartas de los generales Tirso González y Miguel Ángel Tamburini para la provincia del Paraguay”. IHS, Antiguos jesuitas en Iberoamérica, 1, pp. 248321.

Pérez Alonso, Manuel Ignacio (intr. y notas) (1987), Eusebio Francisco Kino, S.J. Cartas a la procura de misiones. México: Universidad Iberoamericana, 1987.

Pérez de Ribas, Andrés (1992), Historia de los triumphos de nuestra santa fee entre las gentes más bárbaras y fieras del Nuevo Orbe (1645). México: Siglo XXI Editores, edición Facsimilar.

Ponce Alcocer, María Eugenia Patricia (Sel. e intr.) (2008), Cartas de la Nueva Francia de los misioneros jesuitas. Siglo XVII. México: Universidad Iberoamericana.

Redden, Andrew (2012), “A las buenas personas no les queda más que el dolor y el gemido’: la violencia del martirio y la violencia del mártir en las fronteras de Nueva España y Paraguay”. En: Zazueta M. José Carlos (ed.), Seminario La religión y los jesuitas en el Noroeste Novohispano, Vol. V. Culiacán: El Colegio de Sinaloa.

Rodríguez, Miguel Ángel (Ed.) (1991), Monumenta Mexicana. Roma: IHSI, vol. 8. 
Zermeño, Guillermo (Sel. e intr.) (2008), Cartas edificantes y curiosas de algunos misioneros jesuitas del siglo XVIII. Travesías, itinerarios, testimonios. México: Universidad Iberoamericana.

Zubillaga, Félix (ed.) (1969), "La provincia jesuítica de Nueva España. Su fundamento económico siglo XVI". Archivum Historicum Societatis Iesu 38, pp. 3-169.

- (1956), Monumenta Mexicana. Roma: IHSI, vols. I.

- (1959), Monumenta Mexicana. Roma: IHSI, vol. II.

- (1968), Monumenta Mexicana. Roma: IHSI, vol. III.

(1971), Monumenta Mexicana. Roma: IHSI, vol. IV.

- (1973), Monumenta Mexicana. Roma: IHSI, vol. V.

-(1976), Monumenta Mexicana. Roma: IHSI, vol. VI.

- (1981), Monumenta Mexicana. Roma: IHSI, vol. VII.

136 Gilberto López Castillo. De la "ciudad eterna" a las "misiones más remotas"... 121-136. 